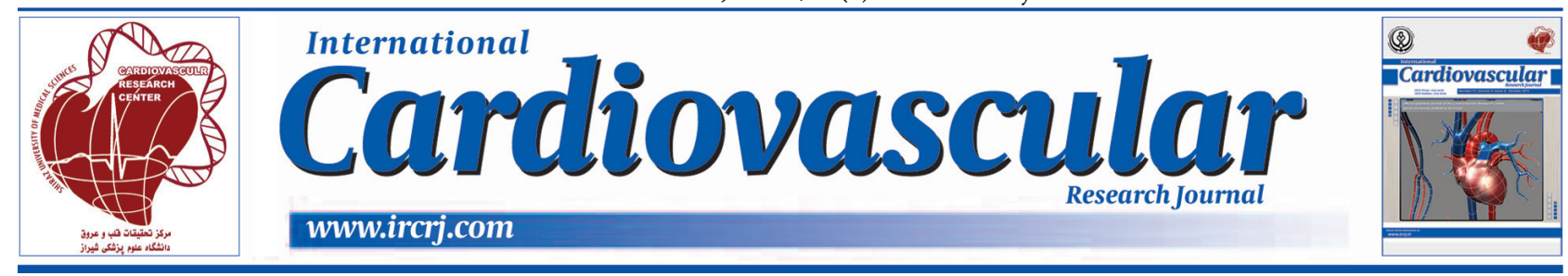

\title{
EP Testing in Asymptomatic Brugada Patients with Recurrent Palpitations. Just do it!
}

\author{
Filippo Maria Cauti ${ }^{1,2}$, Andrea Radinovic ${ }^{1}$, Patrizio Mazzone ${ }^{1, *}$, Damiano Regazzoli ${ }^{1}$, Fabrizio \\ Guarracini ${ }^{1}$, Simone Sala ${ }^{1}$, Marco Ancona ${ }^{1}$, Nicola Trevisi ${ }^{1}$, Paolo Della Bella ${ }^{1}$
}

${ }^{1}$ Arrhythmia Unit and Electrophysiology Laboratories, San Raffaele Hospital, Milano, Italy
${ }^{2}$ Department of Clinical and Molecular Medicine, Faculty of Medicine and Psychology, University of Rome Sapienza, Rome, Italy

\begin{tabular}{l}
\hline A R T I C L E I N F O \\
\hline Article Type: \\
Case Report \\
\hline
\end{tabular}

Article History:

Received: 07 Jan 2015

Revised: 13 Mar 2015

Accepted: 08 Apr 2015

\begin{abstract}
A B S T R A C T
Brugada syndrome is a rare channelopathy characterized by an increased risk of sudden cardiac death. Patients with confirmed Brugada pattern and a history of palpitations without major events should be scheduled for risk stratification. Herein, we reported a patient with type I Electrocardiogram (ECG) pattern induced at the Ajmaline test and a family history of sudden cardiac death who had episodes of palpitations. We, therefore, described our flowchart in order to assess his sudden cardiac death risk.
\end{abstract}

Keywords:

Brugada Syndrome

Ventricular Tachycardia

Electrophysiology

-Implication for health policy/practice/research/medical education:

Our case report clearly shows how stimulation should include both a ventricular and atrial protocol in patients with Brugada syndrome who undergo an EP study. This approach will help stratify the risk and treat the potential supraventricular tachycardia associated with this syndrome.

\section{Introduction}

Brugada syndrome is a rare heritable arrhythmogenic disease characterized by a coved ST segment elevation in the right precordial leads and by an increased risk of sudden cardiac arrest as a result of polymorphic ventricular tachyarrhythmias or ventricular fibrillation (1). Although implantable cardioverter defibrillators are considered to be the main therapy in patients with a history of ventricular arrhythmias or syncope, risk stratification in cases without these symptoms remains controversial. Patients with confirmed Brugada pattern and a history of palpitations without major events should be scheduled for risk stratification. Even though its value has been questioned, inducibility of VTs/VF at programmed electric stimulation is widely used to select

*Corresponding author: Patrizio Mazzone, Arrhythmia Unit and Electrophysiology Laboratories, San Raffaele Hospital, Via Olgettina 60, Milan 20132, Italy, Tel: +39-02264336896;

E-mail: mazzone.patrizio@hsr.it candidates to receive a prophylactic implantable cardiac defibrillator in these categories.

Herein, we report a patient with type I Electrocardiogram (ECG) pattern induced at the Ajmaline test and a family history of sudden cardiac death who had episodes of palpitations. We describe and discuss our management proving the importance of complete electrophysiological testing in the setting of Brugada asymptomatic patients' risk stratification.

\section{Case Presentation}

A 65-year old man with hypertension and dyslipidemia was admitted to our department due to recurrent episodes of palpitations never documented by ECGs. Of note, the frequency of the episodes had increased remarkably during the last year. The patient had a history of Brugada type I ECG induced by an Ajmaline test several years ago. He also had a recent family history of sudden cardiac death; his brother died suddenly after an episode of fever at the 
age of 61 while cycling, no autopsy was performed. The patient was under aspirin $100 \mathrm{mg}$, atorvastatin $10 \mathrm{mg}$, and zofenopril $30 \mathrm{mg}$.

The ECG at admission demonstrated sinus rhythm with mild saddle back like ST elevation, mainly evident in lead V1 - V2 with no QRS fragmentation (Figure 1). Physical examination and laboratory tests were unremarkable. The echocardiogram also showed a structurally and functionally normal heart. During ECG monitoring, no arrhythmias were documented. On the basis of the history, the patient underwent an electrophysiological study in order to identify a correlation to his symptoms and to clarify his risk.

No episodes of VTs/VF were induced with up to three ventricular extrastimuli (double site stimulation) under basal condition. Ventricular refractory period was $>200$ msec. After a programmed atrial stimulation protocol, a well-tolerated, clinical, Atrioventricular Nodal Reentrant Tachycardia (AVNRT) was induced (Figure 2). The AVNRT was successfully treated with radiofrequency catheter ablation of the slow pathway. Since the patient had no history of syncope, had no VT/VF inducibility at the EP testing, and all the symptoms were consistently related due to AVNRT, we considered the patient to be at low risk. After receiving his written informed consent, blood samples were taken in order to screen SCNA5 genes. The patient was discharged in good clinical conditions. All the family members were called to undergo an evaluation and were closely followed up. After three months of close follow-up, the patient had no episodes of palpitation. ECG Holter monitoring also demonstrated no evidence of arrhythmia or conduction disturbances; SCN5A test was negative.

\section{Discussion}

In the current report, we presented a patient with Brugada type I ECG pattern (provoked by a Class I sodium channel blocker) and a history of tachycardia who developed AVNRT at an electrophysiological study during his risk stratification in our EP laboratories.

The association between Brugada syndrome and Supraventricular Tachycardia (SVT) was first described in 2001 (2). A recent multicenter study demonstrated that $23 \%$ of Brugada patients presented with SVT, including AVNRT (7\%), atrioventricular tachycardia (2\%), atrial tachycardia (3\%), and atrial fibrillation or flutter (11\%) (3).

Although the pathophysiological basis of the occurrence

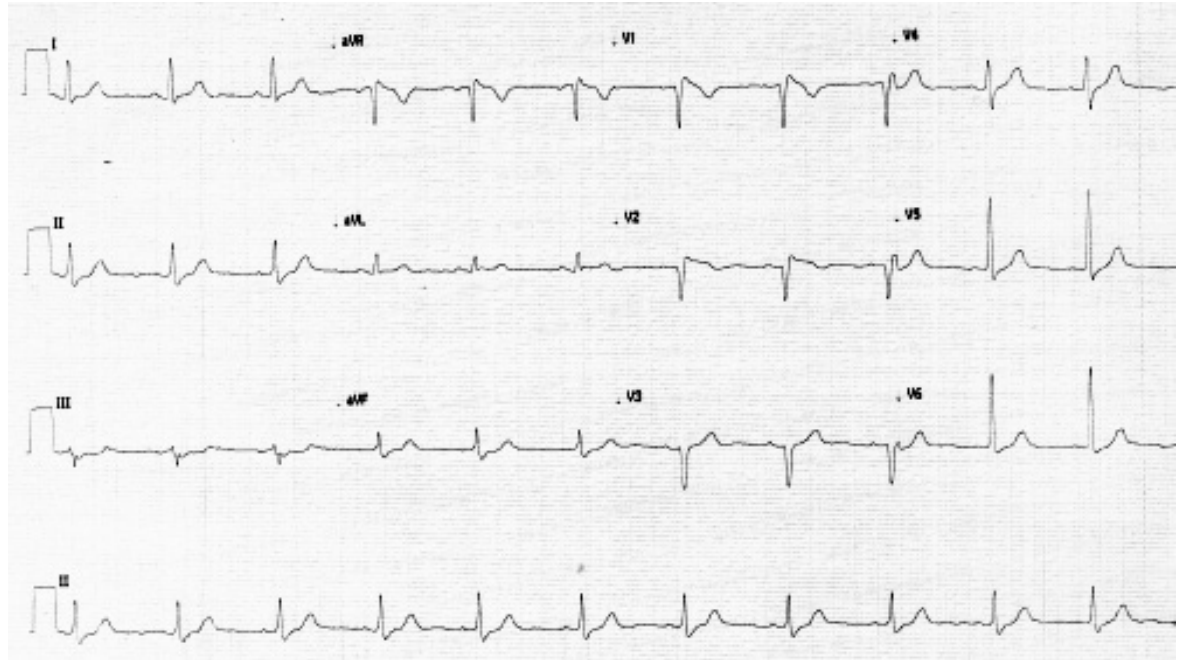

Figure 1. 12-Lead Basal ECG

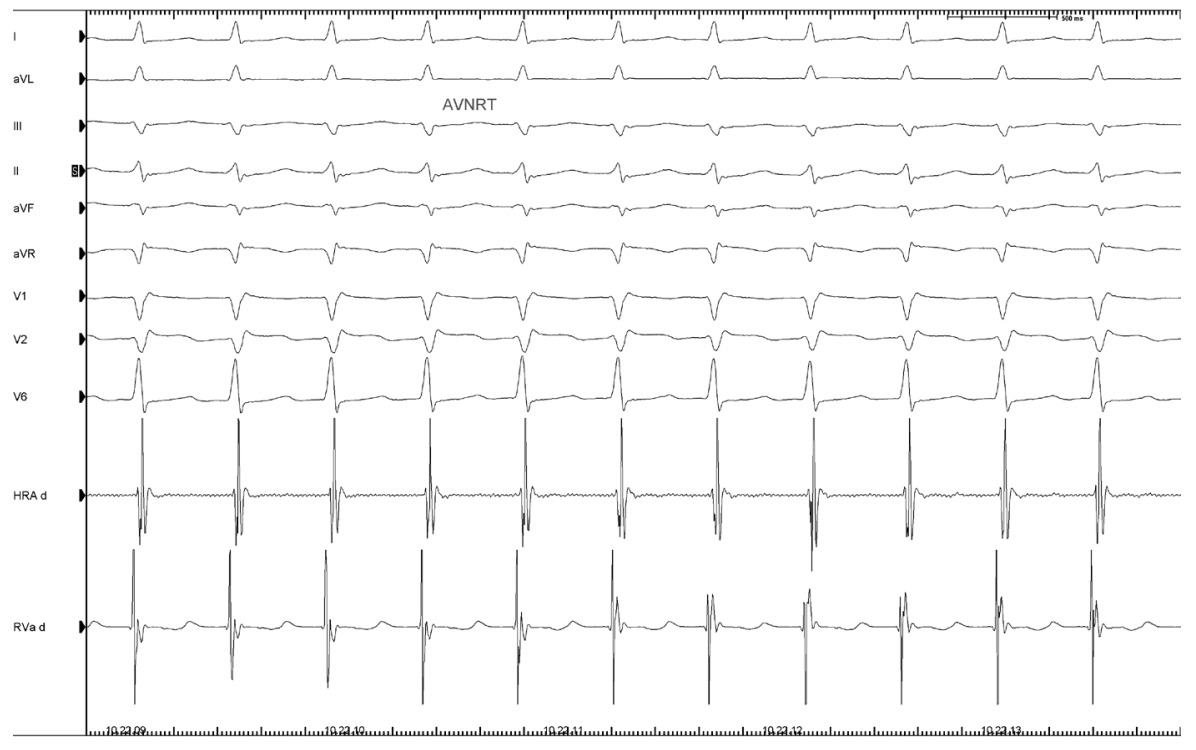

Figure 2. Atrioventricular Nodal Reentrant Tachycardia 
of SVT in Brugada patients in not known, one could speculate that the heterogeneity in repolarization found in the ventricles that occurs in the Brugada syndrome is also extended to the atria and the atrioventricular node (2). Theoretically, involvement of the perinodal atrial tissue with increase of conduction times may lead to two pathways with the propensity to AVNRT (4).

The most challenging aspect of Brugada syndrome is the lack of robust recommendations for management of individuals presenting with electrocardiographic diagnosis of the disease without a previous documentation of arrhythmias. The main missing element to formulate recommendations for managing these patients is the lack of agreement on the annual incidence of life-threatening arrhythmias at follow-up and the discordant opinions on the value of VTs/VF inducibility to identify patients at a higher risk of cardiac arrest (5-12).

Overall, considering the published data regarding SVT, we believe that if a patient with asymptomatic Brugada syndrome performs an EP study for risk stratification, a complete protocol should be performed to exclude any other form of treatable arrhythmias. Moreover, in patients with Brugada syndrome who undergo an EP study, stimulation should include both ventricular and atrial protocols. This approach will help stratify the risk and treat the potential SVT associated with this syndrome.

\section{Acknowledgements}

There is no acknowledgement.

\section{Authors' Contribution}

Study concept and design: Filippo Maria Cauti and Patrizio Mazzone; Drafting of the manuscript: Andrea Radinovic and Filippo M. Cauti; Critical revision of the manuscript for important intellectual content: Patrizio Mazzone and Paolo Della Bella; Study supervision: All the authors

\section{Funding/Support}

There is no funding/support.

\section{Financial disclosure}

There is no financial disclosure.

\section{References}

1. Brugada P, Brugada J. Right bundle branch block, persistent ST segment elevation and sudden cardiac death: a distinct clinical and electrocardiographic syndrome. A multicenter report. J Am Coll Cardiol. 1992;20(6):1391-6.

2. Eckardt L, Kirchhof P, Loh P, Schulze-Bahr E, Johna R, Wichter T, et al. Brugada syndrome and supraventricular tachyarrhythmias: a novel association? J Cardiovasc Electrophysiol. 2001;12(6):680-5.

3. Schimpf R, Giustetto C, Eckardt L, Veltmann C, Wolpert C, Gaita $\mathrm{F}$, et al. Prevalence of supraventricular tachyarrhythmias in a cohort of 115 patients with Brugada syndrome. Ann Noninvasive Electrocardiol. 2008;13(3):266-9.

4. Chen J, Josephson ME. Atrioventricular nodal tachycardia occurring during atrial fibrillation. J Cardiovasc Electrophysiol. 2000;11(7):812-5.

5. Antzelevitch C, Brugada P, Borggrefe M, Brugada J, Brugada R, Corrado D, et al. Brugada syndrome: report of the second consensus conference: endorsed by the Heart Rhythm Society and the European Heart Rhythm Association. Circulation. 2005;111(5):659-70.

6. Brugada P, Brugada R, Brugada J. Should patients with an asymptomatic Brugada electrocardiogram undergo pharmacological and electrophysiological testing? Circulation. 2005;112(2):279-92; discussion -92 .

7. Brugada P, Brugada R, Mont L, Rivero M, Geelen P, Brugada J. Natural history of Brugada syndrome: the prognostic value of programmed electrical stimulation of the heart. $J$ Cardiovasc Electrophysiol. 2003;14(5):455-7.

8. Delise P, Allocca G, Marras E, Giustetto C, Gaita F, Sciarra L, et al. Risk stratification in individuals with the Brugada type 1 ECG pattern without previous cardiac arrest: usefulness of a combined clinical and electrophysiologic approach. Eur Heart J. 2011;32(2):169-76.

9. Gehi AK, Duong TD, Metz LD, Gomes JA, Mehta D. Risk stratification of individuals with the Brugada electrocardiogram: a meta-analysis. J Cardiovasc Electrophysiol. 2006;17(6):577-83.

10. Priori SG, Gasparini M, Napolitano C, Della Bella P, Ottonelli AG, Sassone B, et al. Risk stratification in Brugada syndrome: results of the PRELUDE (PRogrammed ELectrical stimUlation preDictive valuE) registry. J Am Coll Cardiol. 2012;59(1):37-45.

11. Probst V, Veltmann C, Eckardt L, Meregalli PG, Gaita F, Tan HL, et al. Long-term prognosis of patients diagnosed with Brugada syndrome: Results from the FINGER Brugada Syndrome Registry. Circulation. 2010;121(5):635-43.

12. Zipes DP, Camm AJ, Borggrefe M, Buxton AE, Chaitman B, Fromer M, et al. ACC/AHA/ESC 2006 guidelines for management of patients with ventricular arrhythmias and the prevention of sudden cardiac death: a report of the American College of Cardiology/ American Heart Association Task Force and the European Society of Cardiology Committee for Practice Guidelines (Writing Committee to Develop guidelines for management of patients with ventricular arrhythmias and the prevention of sudden cardiac death) developed in collaboration with the European Heart Rhythm Association and the Heart Rhythm Society. Europace. 2006;8(9):746-837. 